\title{
Study Overall Status Study Stopped Reason Code
}

National Cancer Institute

\section{Source}

National Cancer Institute. Study Overall Status Study Stopped Reason Code. NCI

Thesaurus. Code C94099.

A coded value specifying why the study has been halted or terminated (for suspended, terminated or withdrawn studies). 